\title{
Technologies for fast blue and IR PIN diodes
}

\author{
Detlef Sommer, Dr.Konrad Bach
}

X-FAB Semiconductor Foundreis AG

Haarbergstr. 67, 99097 Erfurt, Germany

\section{Requirements of Photo Detector Integrated Circuits}

Photo detector integrated circuits (PDIC) require high-sensitivity and high-bandwidth photo diodes for the latest generation of Blu-ray data storage devices. Due to the very short $405 \mathrm{~nm}$ wavelength used, carriers are generated close to the surface. Standard photo diodes have only a low sensitivity for blue light. Therefore, special adapted photo diodes are necessary to support sensitivity higher than $0.25 \mathrm{~A} / \mathrm{W}$ for a $405 \mathrm{~nm}$ wavelength. In addition, legacy DVDs and CDs must be supported DVDs with a $650 \mathrm{~nm}$ wavelength for red laser diodes, and CDs with a $750 \mathrm{~nm}$ infrared wavelength. Thus the required sensitivities fall between $0.4 \mathrm{~A} / \mathrm{W}$ and $0.45 \mathrm{~A} / \mathrm{W}$.

Although the latest generation of Blu-ray data storage devices supports $6 x$ to $8 x$ speeds, nextgeneration devices will need $12 x$ speed. Increased rotation speed requires increased bandwidth for all components of the receiving part of a data storage system. The total system bandwidth of $300 \mathrm{MHz}$ requires $600 \mathrm{MHz}$, including a margin for the receiving photo diode. The maximum DVD speed of $24 \mathrm{x}$ requires a diode bandwidth of $400 \mathrm{MHz}$, while the maximum 52x rotation speed for CDs requires $5 \mathrm{MHz}$.

\section{High Sensitivity due to Optical Window Etching and Anti Reflecting Coating}

Due to the standard silicon process, several isolation and passivation layers are deposited on top of a photo diode. This stack on top of the photo diode reduces light transmission. The allowed variation of the stack is in the range of $1 / 4$ of the light wavelength; it can cause unpredictable losses for dedicated wavelengths, due to interference at the dielectric layer interfaces.
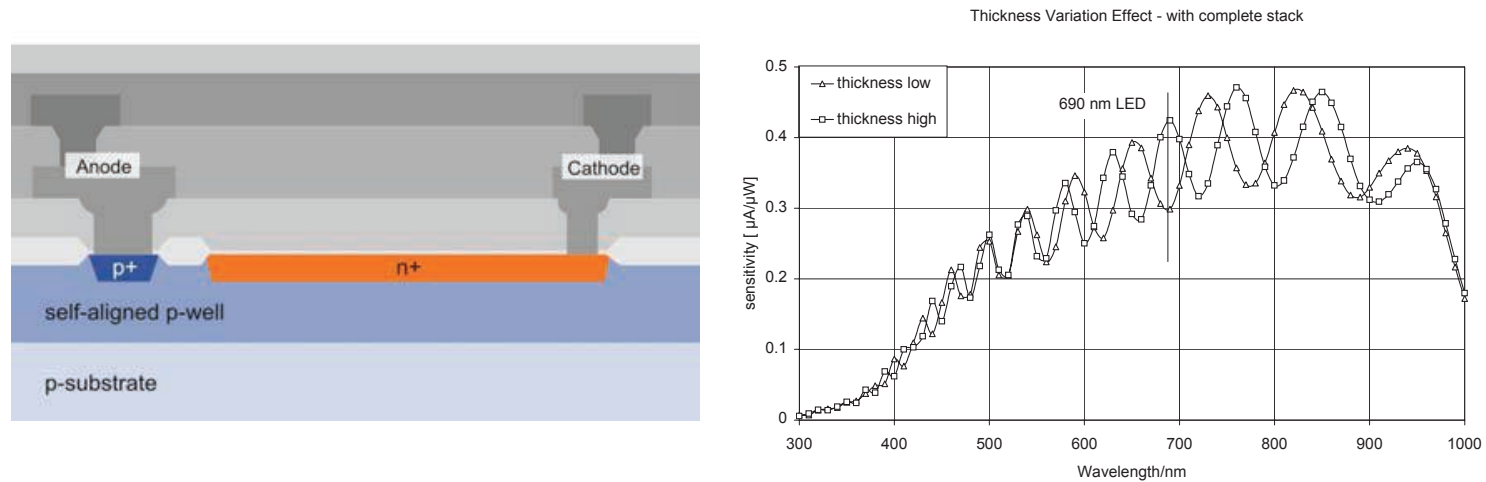

Figure 1: Material on top of the diode reduces transmission. Allowed variation of the stack causes uncontrolled interferences.

A highly sensitive photo diode needs high transmission. The etching away of the material on top increases the transmission by giving light photons direct access to the diode surface. Even the isolator stack on top of the photo diode can be very thick (up to $7 \mu \mathrm{m}$ for a four-metal design); the etching stop on top of the photo diode must be very accurate. The surface should not be damaged, because blue is detected close to surface and damages would cause carrier recombination (lower 
sensitivity) and increased leakage (noise). A special solution for a soft-landing etching has been developed and is patent-pending.

Unpredicted interference losses are reduced by applying an anti-reflective coating (ARC). The ARC layer thickness is adjusted to support a maximum sensitivity for the Blu-ray wavelength of $405 \mathrm{~nm}$. The ARC layer also protects the surface of the photo diode chemically and mechanically.

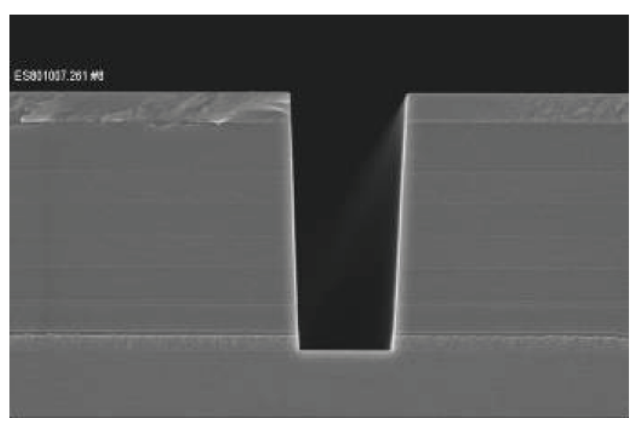

Figure 2: Optical window etching removes the isolator stack on top of the diode and provides direct access to the surface.

\section{Triple Wavelength Photo Diode}

Short wavelengths such as blue light are detected close to the surface of a photo diode. The main part of photo carriers is generated in the first $100 \mathrm{~nm}$, posing some challenges for designing a bluesensitive photo diode in order to get maximum sensitivity. The surface may act as a recombination plane where carriers get lost. Also the quite high doping usually applied for shallow source drain junctions causes recombination. Shallow junctions, manufactured by ion implantation, often comprise secondary implant defects that have no drawbacks for the unipolar MOSFETS but act as recombination centers for the important minority photo carriers.

Available lower doped areas not subject to the drawback of the doping-related recombination often have retrograde profiles. These build a "retarding" electrical field for the photo carriers and cause sensitivity loss and speed reduction.

$\mathrm{X}-\mathrm{FAB}$ has developed a special technique to maintain a very low surface recombination velocity in combination with an optimum cathode doping profile.

Its approach leads to a quantum efficiency of more than $90 \%$ for the blue light $(405 \mathrm{~nm})$.

Although the carriers generated by red and IR must be captured deeper in the material, they also benefit from the improved surface properties and tailored cathode profile. Therefore, X-FAB's photo diode can be considered as a "triple wavelength photo diode," showing a high sensitivity of approximately $0.3 \mathrm{~A} / \mathrm{W}$ or $92 \%$ quantum efficiency for blue light of $405 \mathrm{~nm}$. The sensitivities for DVDs $(650 \mathrm{~nm})$ and $\mathrm{CDs}(780 \mathrm{~nm})$ are still high at $0.44 \mathrm{~A} / \mathrm{W}$ and $0.4 \mathrm{~A} / \mathrm{W}$ respectively. 


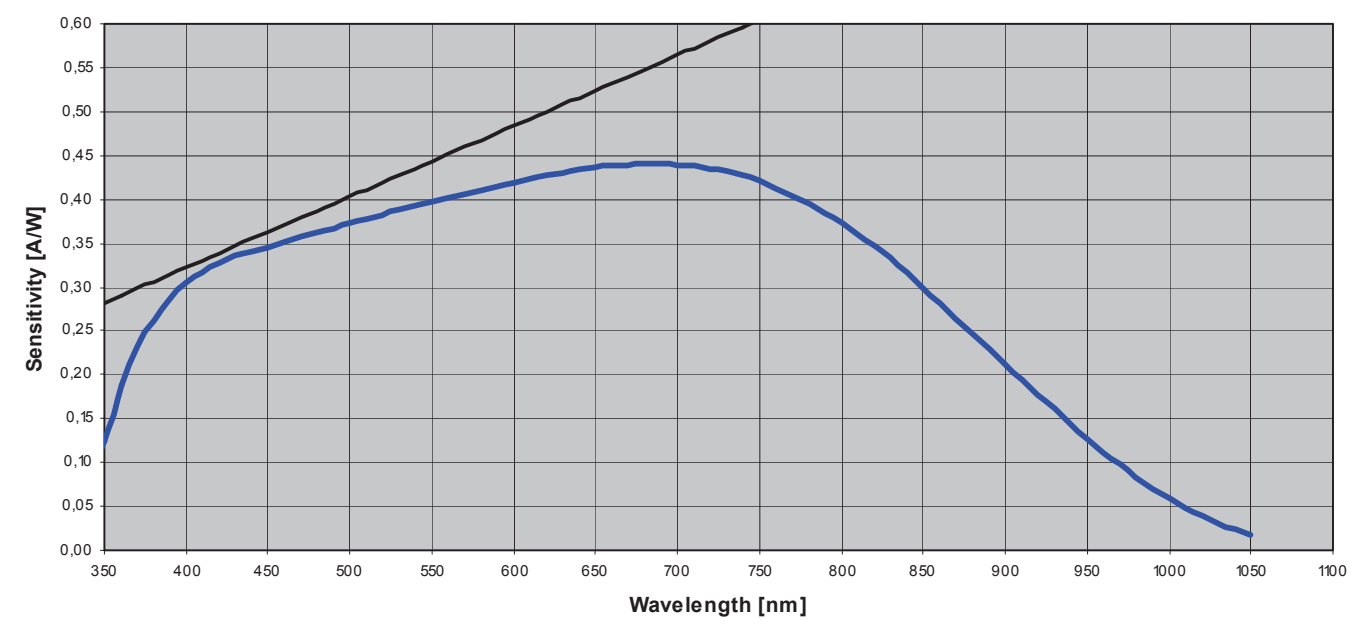

Figure 3: Triple wavelength PIN Diode. High sensitivity at 405nm (Blu-ray) has QE of $92 \%$ resulting in $0.3 A / W$. Sensitivity for $650 \mathrm{~nm}$ (DVD) is $0.44 \mathrm{~A} / \mathrm{W}$ and $780 \mathrm{~nm}$ (CD) is $\sim 0.4 \mathrm{~A} / \mathrm{W}$.

\section{PIN Diode approach for high bandwidth}

A PIN diode has been developed to support these high bandwidth requirements..

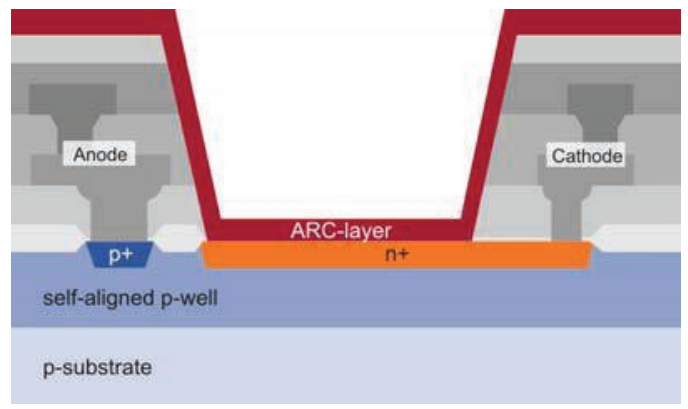

Figure 4: Cross section of PIN photo diode.

In the normal CMOS-well approach (i.e. p-wafer with n-well and quasi p-well) a high ohmic low doped epitaxial wafer material is used $(1 \mathrm{e} 13 / \mathrm{cm} 3 \mathrm{vs} .1 \mathrm{e} 15 / \mathrm{cm} 3)$. A dedicated cathode implant is added, forming a deep depletion zone in the substrate. Due to the high electrical field, most carriers drift and only a few diffuse. Photo detection becomes very fast, and CMOS behaviour does not degrade.

The bandwidths reached are $680 \mathrm{MHz}$ for Blu-ray, $670 \mathrm{MHz}$ for DVD and $150 \mathrm{MHz}$ for $C D$, respectively. 


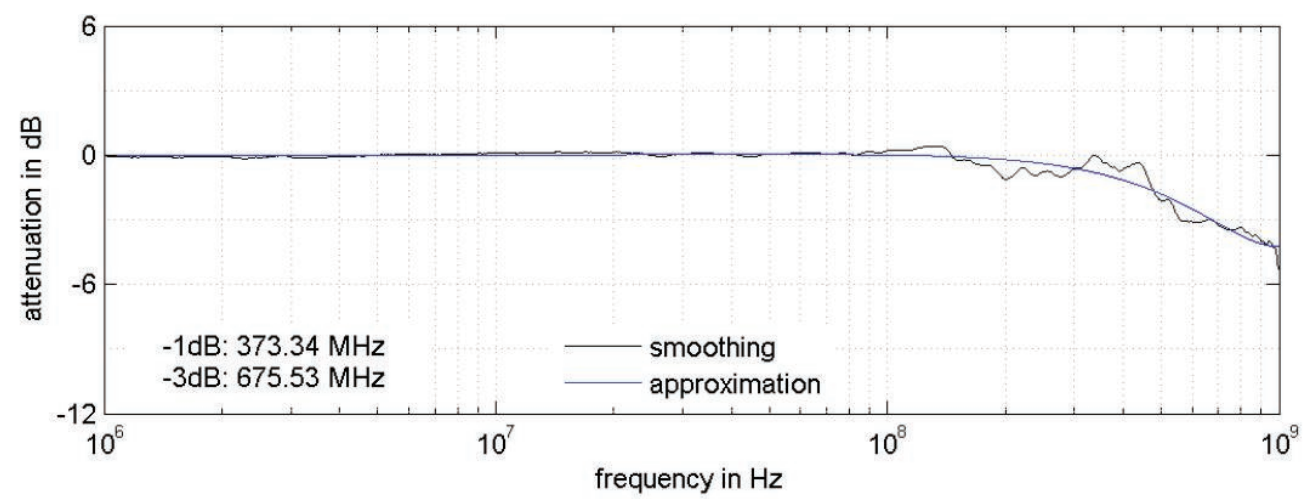

Figure 5: Bandwidth of PIN photo diode at a wavelength of $405 \mathrm{~nm}$.

\begin{tabular}{|c|c|c|}
\hline Standard & $\begin{array}{c}\text { Wavelength } \\
{[\mathbf{n m}]}\end{array}$ & $\begin{array}{c}\text { Bandwidth - 3dB } \\
{[\mathbf{M H z}]}\end{array}$ \\
\hline Blu-ray & 405 & 680 \\
\hline DVD & 650 & 670 \\
\hline CD & 780 & 150 \\
\hline
\end{tabular}

Table 1: Bandwidth of triple wavelengths PIN photo diode.

\section{PIN Diode Modelling}

A PDIC includes additional critical components besides the PIN diode. Optimizing the interfaces and the components themselves requires significant simulation effort. A model of the PIN diode is supported for simulation. The PIN photo diode as a sensor element is implemented in the "electrical" design flow. It is defined as a three-terminal device. In addition to the anode and cathode pin of a standard diode, a third "lightpower" pin is included in the model. The wavelength can be used as a parameter. Spectrum simulation can be done by a parameter sweep.

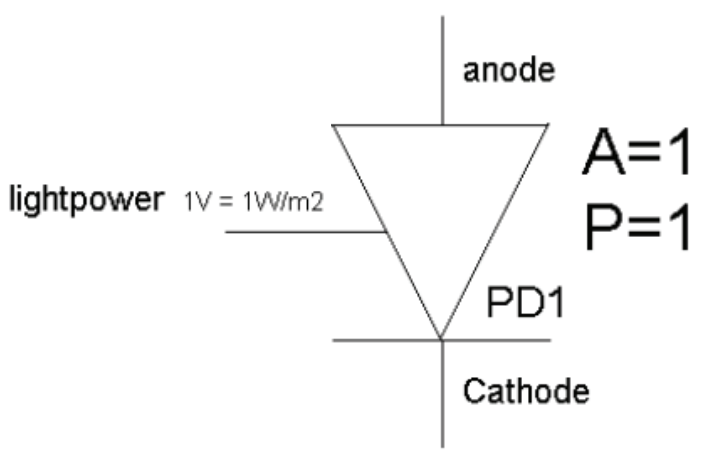

Figure 6: Schematic of PIN photo diode model. 
Simulation, design rule check (DRC) and back annotation can be done using the standard design flow. The accuracy of the model for sensitivity and bandwidth has been silicon-proven by measurement.

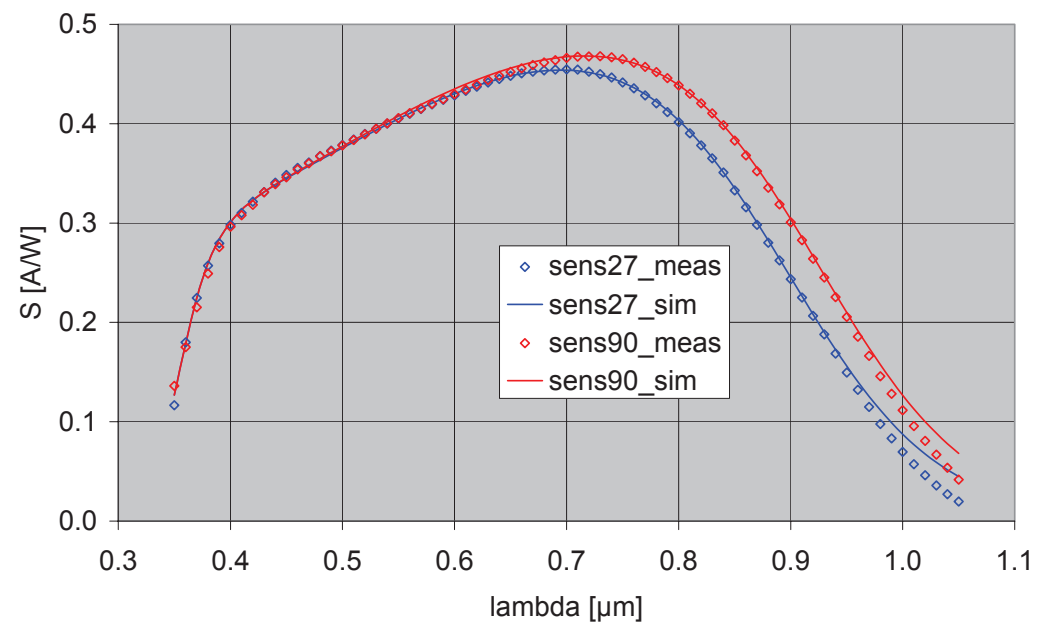

Figure 7: PIN diode comparison simulated versus measured sensitivity at $27^{\circ} \mathrm{C}$ and $90^{\circ} \mathrm{C}$.

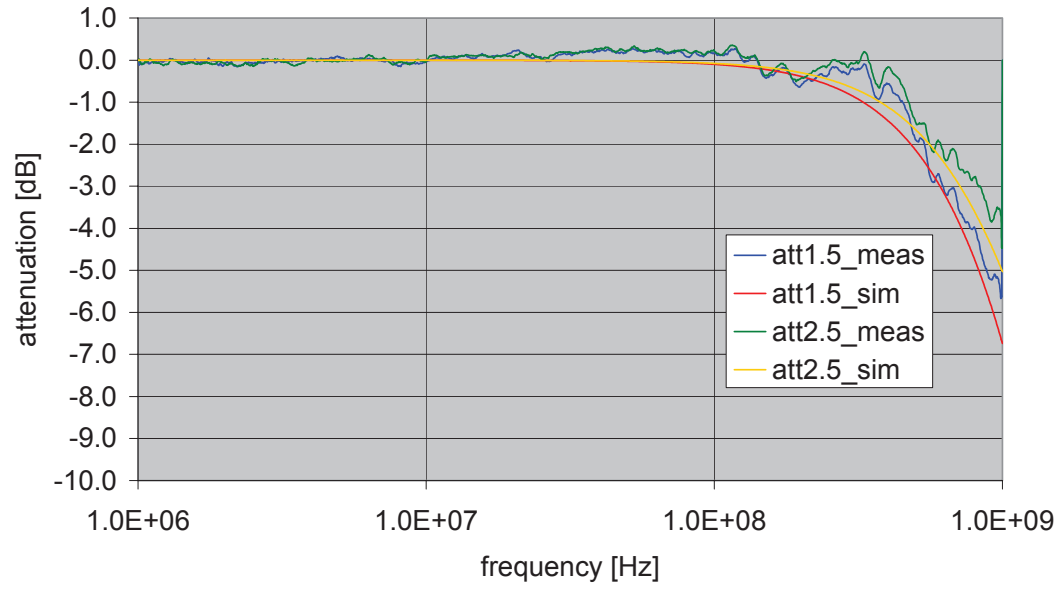

Figure 8: PIN diode comparison simulated versus measured frequency response $(100 \mu \mathrm{m} \times 100 \mu \mathrm{m} ; 405 \mathrm{~nm} ; \mathrm{Vr}=1.5 \mathrm{~V}, 2.5 \mathrm{~V})$. 


\section{Summary}

X-FAB has developed a PIN diode on its $0.35 \mu \mathrm{m}$ technology platform that fulfils all requirements of a triple wavelength photo diode for Blu-ray, DVD and CD. The PIN diode supports sensitivity and bandwidth for $12 x$ speed Blu-ray applications.

A special optical window etched on top of the photo diode and the deposition of an Anti-Reflecting Coating optimized for the Blu-ray wavelength of $405 \mathrm{~nm}$ increases the sensitivity to $0.3 \mathrm{~A} / \mathrm{W}$, resulting in a quantum efficiency of $92 \%$.

As there is still a high sensitivity for DVDs $(650 \mathrm{~nm})$ with $0.44 \mathrm{~A} / \mathrm{W}$ and for CDs $(780 \mathrm{~nm})$ with $0.4 \mathrm{~A} / \mathrm{W}$, the photo diode supports triple-wavelength applications.

A PIN diode solution is used to comply with the high bandwidth requirements. Using low doped wafer material allows for a high-speed photo diode without impacting the CMOS behaviour of the circuit. A special cathode implant reaches a deeper electrical field. Most of the carriers can drift, and only a few diffuse. This means that photo detection becomes very fast to address high-bandwidth applications.

A model of the PIN diode is supported for simulation. The accuracy of the model for sensitivity and bandwidth has been silicon-proven by measurement. 